\title{
CONTROLLING VIRTUAL CLOUDS AND MAKING IT RAIN PARTICLE SYSTEMS IN REAL SPACES USING SITUATED AUGMENTED SIMULATION AND PORTABLE VIRTUAL ENVIRONMENTS
}

\author{
N. Hedley ${ }^{\mathrm{a}, *}$, C. D. Lonergan ${ }^{\mathrm{a}}$ \\ ${ }^{\text {a }}$ Spatial Interface Research Lab, Department of Geography, Faculty of Environment, Simon Fraser University, 8888 \\ University Way, Burnaby, BC Canada V5A 1S6 - (hedley, cd15)@sfu.ca
}

WG II/6

KEY WORDS: mobile augmented reality, situated augmented simulation, geosimulation, gaming, virtual environments, geovisualization

\begin{abstract}
:
The research described in this paper reports on the design, rationale, development and implementation of a set of new geospatial interfaces that combine multi-touch interaction, portable virtual environments, 'geosimulation gaming', and mobile augmented reality. The result is a set of new ways for us to combine the capabilities of geospatial virtual environments, augmented reality and geosimulation. These new hybrid interfaces deliver new geospatial information experiences - new ways of connecting spatial data, simulations, and abstract concepts to real spaces. Their potential to enhance environmental perception and learning must be explored.
\end{abstract}

\section{INTRODUCTION}

\subsection{Background}

Virtual environments have been of interest to geovisualization researchers for some time now. In the past decade, the ability to create high fidelity virtual environments using inexpensive game engine software has unlocked new opportunities for geovisualization researchers. These newer platforms enable us to interact with 3D physics simulations in real time in virtual spaces as individuals and in groups. In the past fifteen years, augmented reality has matured to a point where it now being adopted and deployed by a wide range of user communities in society, industry and research (Azuma, 2001; Hedley et al., 2001; Hedley, 2008).

\subsection{Aims and scope of research}

At the Spatial Interface Research Lab, we developed a set of mobile tangible user interfaces (TUI) to explore new forms of situated geovisualization user experiences that might enhance student perception of the relationship between topography and surface flow in watershed basins. Using 3D physics-based geosimulation and embedded geomatics, we implemented location-aware interfaces that enable students to interactively explore precipitation, watershed topography, and surface flow in everyday spaces. Each separate prototype explores different combinations of: data; geosimulation; geovisualization; interaction design; interface modality; real space; and virtual space.

\subsection{Three groups of interface prototypes developed at SIRL}

Three groups of interface prototypes were designed, developed and implemented, to explore the convergence of the factors mentioned in the previous section.

The first collection of interface prototypes are portable virtual environments that combine geosimulation, geovisualization, and abstract environmental concepts in tangible user interfaces. The second group of interfaces enhance the properties of the first group using device location awareness to show user location within the visualization application. The third set of interface prototypes make it possible to combine geosimulation, geovisualization and abstract concepts with real geographic spaces - in two stages. The first, using situated virtual environments. The second by implementing augmented spaces.

\section{INTERFACE PROTOTYPES}

\subsection{Portable virtual environments}

A Touch of Rain is a multi-modal geospatial interface that combines location, orientation and motion sensor data with tablet touch screen user input capabilities. A tangible 3D simulation space allows users to load a real $3 \mathrm{D}$ digital terrain model of local watersheds, which they can manipulate with their fingertips.

\footnotetext{
* Corresponding author.
} 


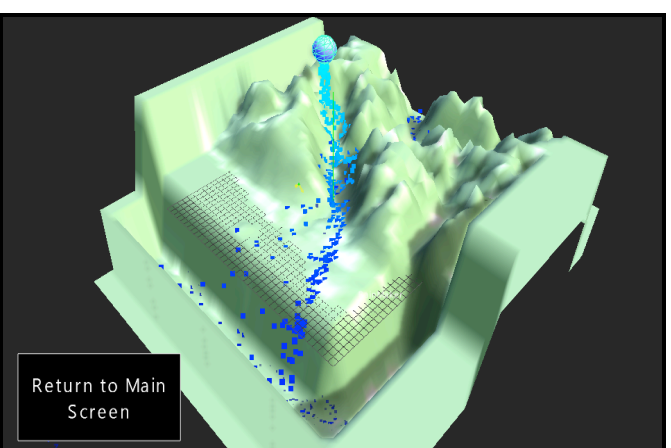

Figure 1. An early Touch of Rain prototype to test the live particle system on the mobile

Instead of focusing on accelerometer-based input, the touch screen is used to provide an interactive watershed simulation. The app consists of a 3D DEM of Vancouver's North Shore Mountains and an interactive rain generator. The user can move the 'raincloud' by touching any part of the terrain so move the generator to that point.

This app has been designed to give the user a real-time rain and watershed simulator. Using a real-time 3D particle system that interacts with a collision map generated from the terrain geometry, users are able to interactively position and move a virtual cloud over the terrain, trigger and control a virtual rainstorm, and see where water particles flow within the topography. This visualization, interface and interaction design enables the user to set up their own simple experiments in a virtual watershed using real data. They can explore informal hypotheses about the flow of precipitation over topography, flowpath alternatives, and simple relative flow quantification.

A MultiTouch of Rain allows multiple virtual clouds and particle systems to operate at once. Each user gets their own cloud. Each cloud has color-coded rain particles. This allows multiple students to engage in collaborative geographic geosimulation gaming, and compare the particle flow outcomes of different precipitation regimes. See figures 2 and 3.

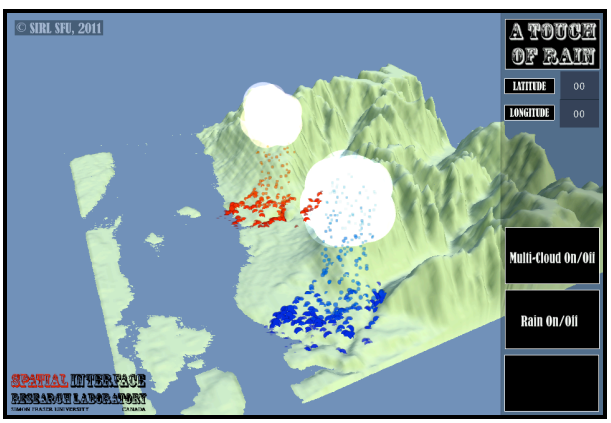

Figure 2. An early A Multi Touch of Rain prototype in twouser mode with a DEM of Vancouver's North Shore mountains.

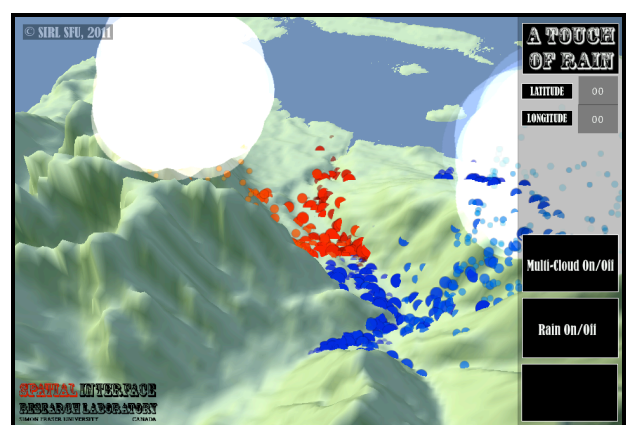

Figure 3. An early A Multi Touch of Rain prototype.

\subsection{Situated virtual environments}

Using the device's coordinates, the basic version of $A$ Situated Touch of Rain is able to display the user's actual location on the digital terrain model. (Fig.3 and 4). This simple capability has considerable potential to connect users to abstract data representations and visualiztion in new ways, by allowing them to see markers or avatars of themselves placed in the virtual scene.

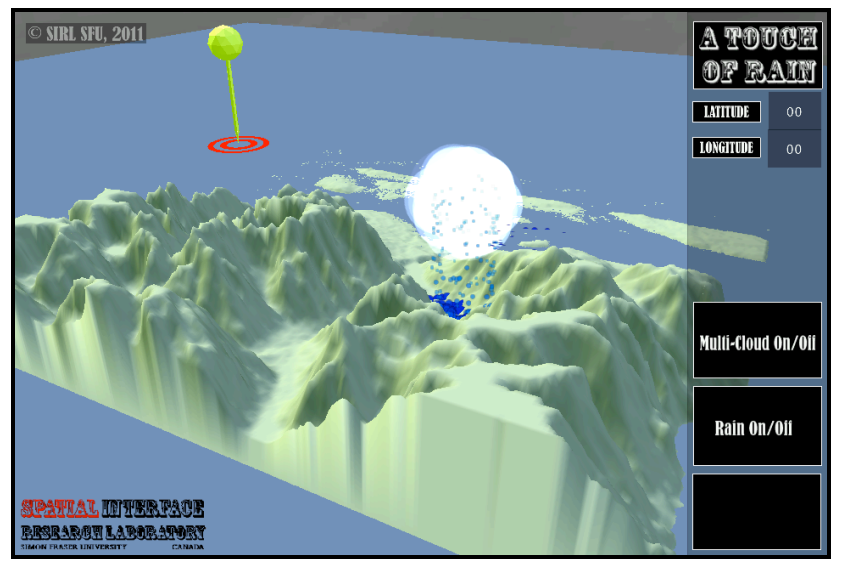

Figure 3. An early prototype of A Situated Touch of Rain in single user mode with a DEM of Vancouver's North Shore mountains. The user's real-time location in the real world is indicated by the yellow stick. Note that when this screen capture was made, we were not physically present in the area covered by the DEM, but on campus - to the Southeast.

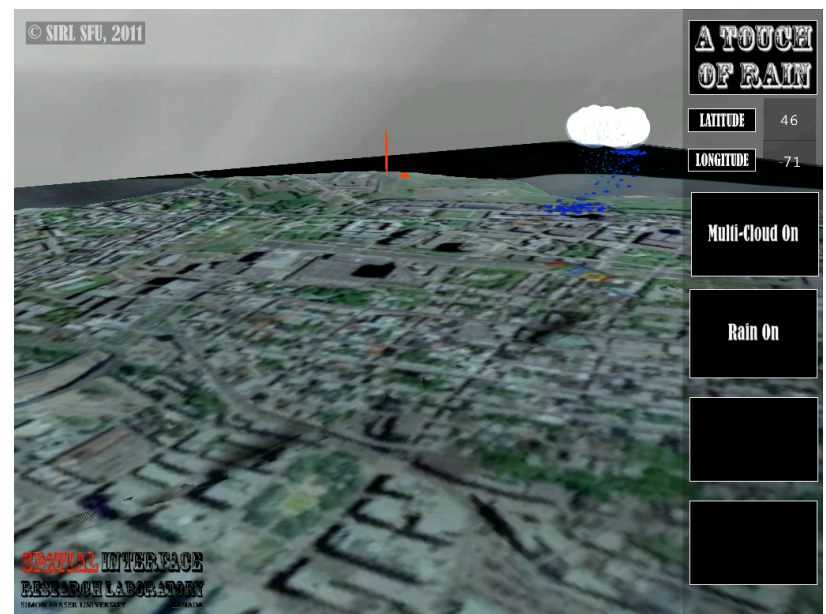

Figure 4. A Situated Touch of Rain in single user mode with orthoimagery overlaid on a DEM. The user's real-time location 
in the real world is indicated by the red stick. Compass orientation is indicated by the red ball.

A more sophisticated version of $A$ Situated Touch of Rain is a situated virtual environment that uses device orientation, acceleration and location sensor data to allow the user to stand in real space, but look into an alternate virtual world in situ. Specifically, this application allows the user to look 'through' location aware mobile devices as portals into parallel virtual geosimulation spaces at the same time as standing on the equivalent real location. (See figure 6).

\subsection{Stepping from situated virtual environments into augmented spaces}

An Augmented Touch of Rain - is a fully mobile augmented reality (MAR) interface that takes this development path one step further. This geovisualization interface allows the user to switch seamlessly between situated virtual environment and situated augmented reality. Users can create, resize and position virtual clouds over topography in a perspective situated virtual space (Fig. 5). They can trigger particle-based rain events from these constructed clouds. These precipitation simulations can be viewed in the same handheld virtual space, or viewed in one of two ways while standing in a real geographic space.

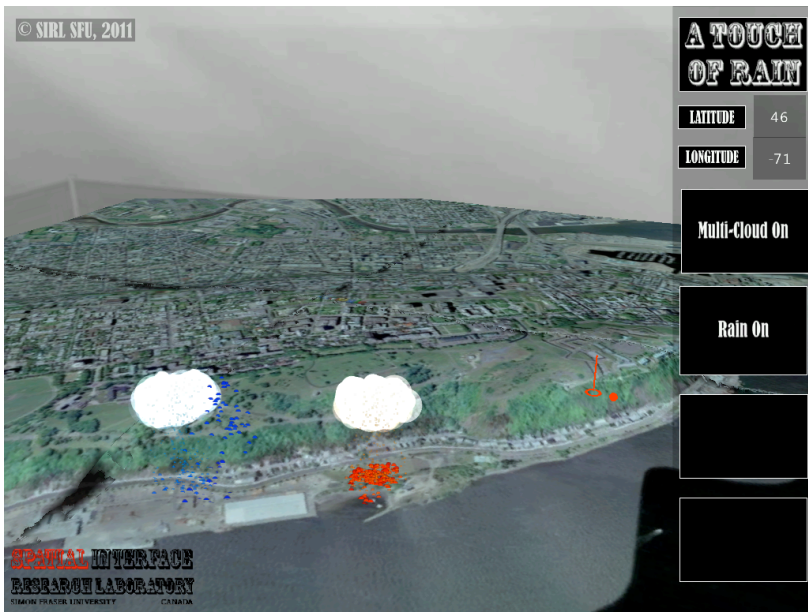

Figure 5. Creating and placing virtual clouds in real space in a location-aware handheld $3 \mathrm{D}$ perspective virtual space. (Note red stick indicating user's location at time of screen capture).

While standing in real geographic space, the user can observe the precipitation simulation falling (or flowing over topography) in either a situated virtual world mode - where they can 'look through' the tablet device, into a situated virtual space that is all around them at one-to-one scale. (See figures 6 and 7).
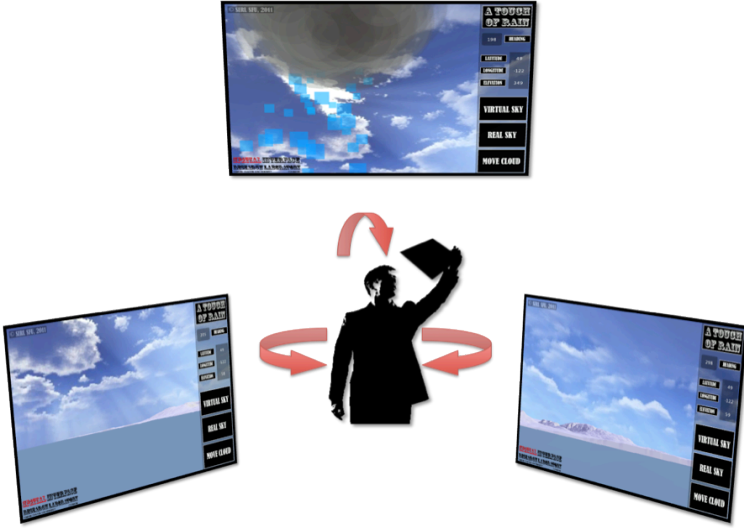

Figure 6. A situated virtual environment interface - using a location-aware, camera-equipped, sensor-enabled tablet to 'look through' a portal into a virtual world at the same scale as the real world. The user can move the tablet to look around at data visualizations of the space they are standing in.

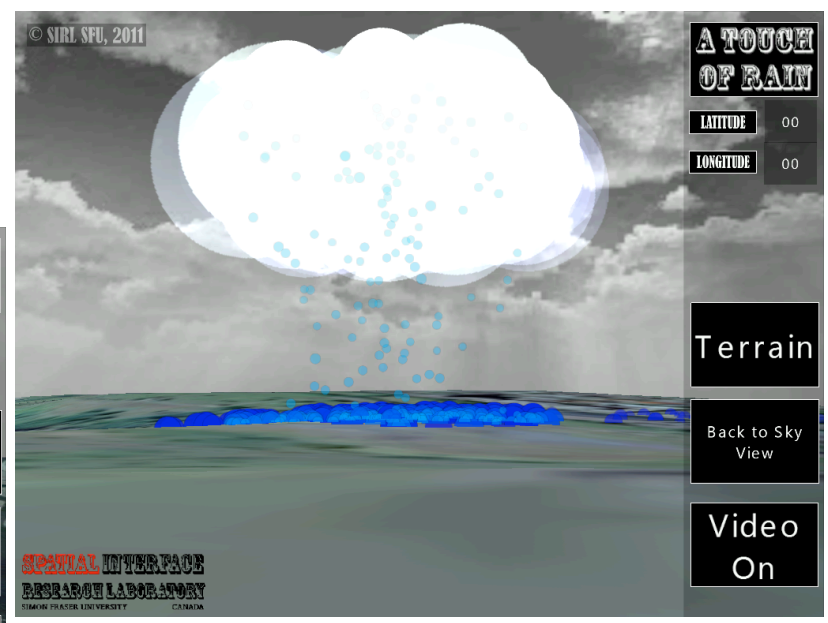

Figure 7. A live screen capture from An Augmented Touch of Rain - showing the user's situated virtual world view.

The other way the user can view and experience the rain simulation, is to view an augmented space. This is the key capability of An Augmented Touch of Rain. After creating the cloud and rain systems seen in figure 5 , and having viewed the situated virtual environment mode (figure 6 and 7), our interface allows the user to selectively toggle components of the virtual world on and off. This results in views that combine real and virtual objects - otherwise known as a 'mixed reality' scene (Milgram and Kishino, 1994). Figure 8 shows a scene from our field testing - with real sky and trees combined with a virtual cloud, virtual rainstorm, and virtual land surface (registered to real coordinates). 


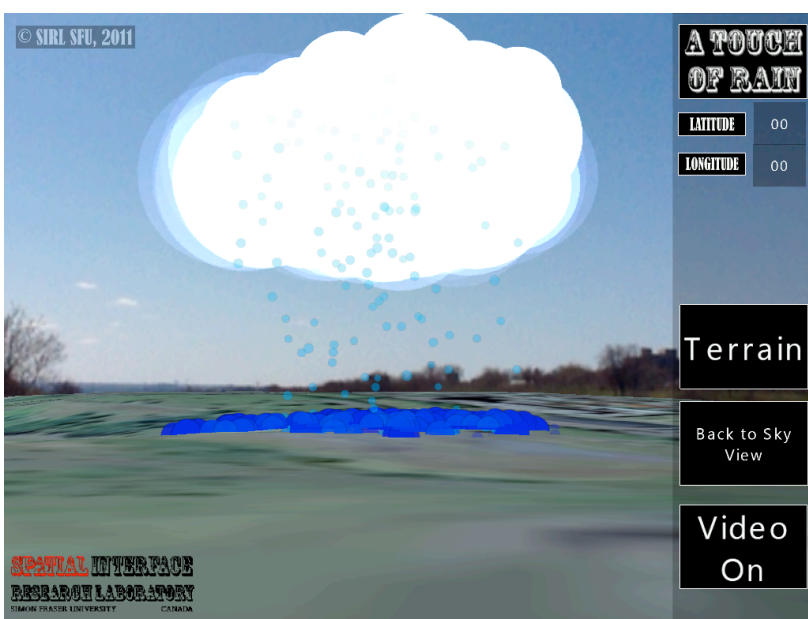

Figure 8. A live screen capture from An Augmented Touch of Rain. The virtual skybox has been toggled off, allowing the user to look around them in the real world through the tablet device and see the virtual clouds that they created. (Note: this is a semi-augmented view, with a virtual topographic land surface that corresponds to reality).

In figure 9 (below), we see another field testing image, where this time the scene is entirely real, apart from a virtual cloud and virtual particle system. But look carefully - the virtual particle system is interacting with the real water surface.

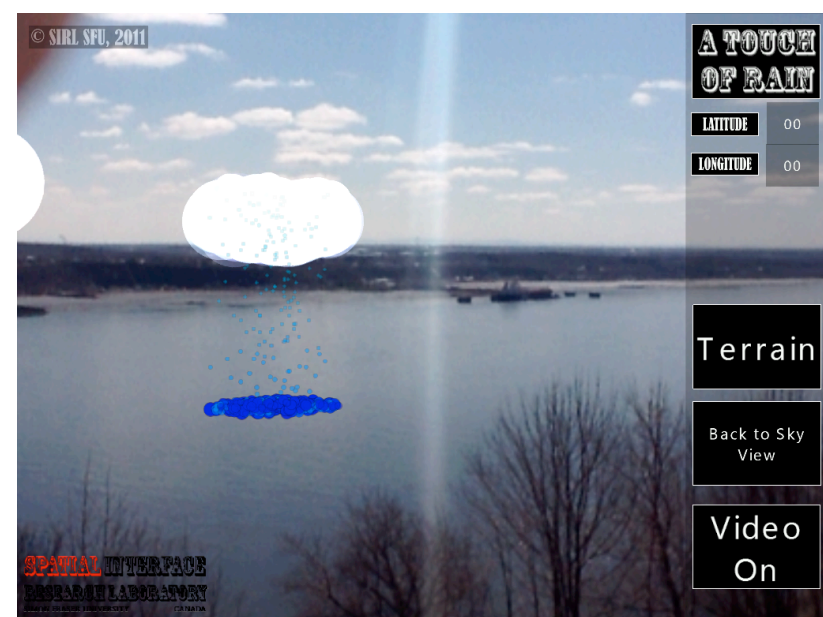

Figure 9. A live screen capture from An Augmented Touch of Rain. The user can look around them at the real world through the tablet device and see the virtual clouds that they created. (Note: this view is more clearly an 'augmented reality' scene).

\subsection{Software and hardware}

We developed these applications and interface prototypes using a mixture of in-house coding and off-the-shelf SDKs integrated with $3 \mathrm{D}$ engine platforms. The specific details will be released at a later date. The applications were designed to run on both Apple iOS and Android operating systems. We have successfully run all of the applications described in this paper on iPhone $3 \mathrm{GS}$, iPhone 4, iPhone 4S, iPad 2 and iPad (3!).

\section{DISCUSSION AND CONCLUSIONS}

Advances in visual, computational, and location-aware capabilities have resulted in increased exploration of mobile devices as legitimate platforms for dispersed, distributed, mobile activities involving spatial information, visualization and interaction. A recent surge in augmented reality uptake in mainstream society has thus far focused primarily on annotating views with the icons of social networks and the 'consumerverse'.

The geospatial community has a unique set of skills which may yet unlock mobile augmented reality's true potential: to see data situated in geographic context; and to see the spatial information all around us. By seeing abstracted data models registered directly to the real geographic environments they attempt to represent, we may provide students with a deeper understanding of their correspondence to methodological decisions in geospatial information use, and complex phenomena in geographic space. To this end, the challenges for geographic researchers, include: to develop robust and elegant ways to connect abstract data representations and real spaces using meaningful visualizations and accessible interfaces. This in turn requires a deeper understanding of interface research and interaction design in a spatial context.

The design, development and implementation of the prototypes briefly described in this paper demonstrate progress in creating mobile AR interfaces that can meaningfully augment geographic spaces with fixed data and dynamic simulations.

However, this research is more than simply exploring new technological frontiers. As we test these prototypes, we are also exploring new relationships between users, spatial information, and real geographic environments. Our field experiences are also yielding new insights in how to design and translate conventional spatial information and visualizations into meaningful objects with which to augment real spaces. This in turn raises compelling new questions about environmental perception, cognition, and the spatial knowledge acquisition that may result.

\section{Copyright}

All images in this paper are (C) Spatial Interface Research Lab, 2011-2012. All rights reserved.

\section{References}

Azuma, R.T., Baillot, Y. Behringer, R., Feiner, S., Julier, S., and B. MacIntyre. (2001). Recent Advances in Augmented Reality. IEEE Computer Graphics and Applications.

Hedley, N.R., Billinghurst, M., Postner, L., and R. May. (2002). Explorations in the use of augmented reality for geographic visualization. Presence: Teleoperators and Virtual Environments, 11(2): 119-133. Cambridge: MIT Press.

Hedley, N., 2008. Real time reification.

P. Milgram and A. F. Kishino, Taxonomy of Mixed Reality Visual Displays IEICE Transactions on Information and Systems, E77-D(12), pp. 1321-1329, 1994.

Thomas, B. H., Close, B., Donoghue, J., Squires, J., De Bondi, P., and Piekarski, W. (2002). First Person Indoor/Outdoor Augmented Reality Application: ARQuake. Personal and Ubiquitous Computing, Vol. 6, No. 2, 2002. 
International Archives of the Photogrammetry, Remote Sensing and Spatial Information Sciences, Volume XXXIX-B2, 2012 XXII ISPRS Congress, 25 August - 01 September 2012, Melbourne, Australia

\section{Acknowledgements}

This research was supported by funding from Canada's

GEOIDE (GEOmatics for Informed DEcisions) Network of

Centres of Excellence (NCE) project PIV-24. 